\title{
The landscape of metabolic brain alterations in Alzheimer's disease
}

Richa Batra ${ }^{*}$, Matthias Arnold ${ }^{2,3^{*}}$, Maria A. Wörheide ${ }^{3}$, Mariet Allen $^{4}$, Xue Wang $^{5}$, Colette Blach $^{2}$, Allan I. Levey ${ }^{6}$, Nicholas T. Seyfried ${ }^{7}$, Nilüfer Ertekin-Taner ${ }^{4,8}$, David A. Bennett ${ }^{9}$, Gabi Kastenmüller ${ }^{3}$, Rima F. Kaddurah-Daouk ${ }^{10 \#}$, Jan Krumsiek ${ }^{1 \#}$

for the Alzheimer's Disease Metabolomics Consortium (ADMC) $)^{11}$

1Department of Physiology and Biophysics, Institute for Computational Biomedicine, Englander Institute for Precision Medicine, Weill Cornell Medicine, New York, NY 10021, USA

${ }^{2}$ Department of Psychiatry and Behavioral Sciences, Duke University, Durham, NC, USA

${ }^{3}$ Institute of Computational Biology, Helmholtz Zentrum München-German Research Center for Environmental Health, 85764 Neuherberg, Germany

${ }^{4}$ Department of Neuroscience, Mayo Clinic Florida, Jacksonville, FL, USA.

${ }^{5}$ Department of Health Sciences Research, Mayo Clinic Florida, Jacksonville, FL, USA.

${ }^{6}$ Goizueta Alzheimer's Disease Research Center, Emory University, Atlanta, GA, USA.

${ }^{7}$ Department of Biochemistry, Emory School of Medicine.

${ }^{8}$ Department of Neurology, Mayo Clinic Florida, Jacksonville, FL, USA.

${ }^{9}$ Rush Alzheimer's Disease Center, Rush University Medical Center, Chicago, IL, USA

${ }^{10}$ Department of Psychiatry and Behavioral Sciences, Duke Institute for Brain Sciences and Department of Medicine, Duke University, Durham, NC, 27708, USA.

${ }^{11}$ The full list of contributing scientists is available at https://sites.duke.edu/adnimetab/team/.

${ }^{*}$ These authors contributed equally to this work

${ }^{\#}$ Corresponding authors

Contact: Jan Krumsiek (jak2043@med.cornell.edu) and Rima Kaddurah-Daouk (rima.kaddurahdaouk@duke.edu) 


\section{Abstract}

We present a comprehensive reference map of metabolic brain changes in Alzheimer's disease (AD). In a multi-center study within the Accelerating Medicines Partnership in AD, we metabolically profiled 500 samples from the dorsolateral prefrontal cortex (DLPFC) and 83 samples from the temporal cortex (TCX). In the DLPFC, 298 metabolites were correlated with ADrelated traits, including late-life cognitive performance and neuropathological $\beta$-amyloid and tau tangle burden. Out of these 298 metabolites, 35 replicated in TCX and a previous study. A conditional analysis suggests that metabolic associations with tangle burden were largely independent of $\beta$-amyloid load in the brain. Our results provide evidence of brain alterations in bioenergetic pathways, cholesterol metabolism, neuroinflammation, osmoregulation, and other pathways. In a detailed investigation of the glutamate/GABA neurotransmitter pathway, we demonstrate how integration of complementary omics data can provide a comprehensive view of dysregulated biochemical processes. All associations are available as an interactive network at https://omicscience.org/apps/brainmwas/. 


\section{Introduction}

Alzheimer's disease (AD) is the most common cause of dementia, with prevalence rates expected to increase markedly over the next decades ${ }^{1}$. It is a neurodegenerative disorder defined by the deposition of $\beta$-amyloid and accumulation of neurofibrillary tangles of phosphorylated tau protein in the brain ${ }^{2}$. These proteinopathies are further accompanied by other pathogenic processes including neuroinflammation, oxidative stress, innate immune response, and neurotransmission ${ }^{1}$. In addition, a large body of evidence implicates metabolic pathways both in the periphery and in the central nervous system in $\mathrm{AD}^{3-8}$. Moreover, metabolic enzymes and transporters are among the most commonly targeted proteins in pharmaceutical interventions across all diseases ${ }^{9,10}$, emphasizing the translational potential of systematically identifying metabolic alterations. However, until now a comprehensive reference map of metabolic brain changes related to $A D$, AD-associated neuropathological manifestation, and cognitive decline has been missing.

Here we present a large, multi-center study from the Accelerating Medicine Partnership in AD (AMP-AD) consortium, analyzing a total of 583 post-mortem brain tissue samples using broad, non-targeted metabolomics measurements. This dataset represents, to the best of our knowledge, the largest metabolomics study of aging brain tissue to date. In the first part of our study, we analyzed 667 metabolites in 500 brain tissue samples from the dorsolateral prefrontal cortex (DLPFC). This resulted in various metabolic associations with AD-related traits, including $\beta$-amyloid and tau tangles neuropathological burden, as well as late-life cognitive performance. We provide preliminary evidence that tau-related pathology is the main driver of metabolic alterations, while $\beta$-amyloid-related alterations are secondary effects. We confirmed a subset of associations in an independent set of 83 temporal cortex (TCX) brain tissue samples, profiled with the same metabolomics platform. In addition, part of our findings overlapped with previously reported $A D$-associated metabolic brain changes ${ }^{5}$. Moreover, we exemplify how the integration of metabolomics with complementary omics data can enable a comprehensive view of biochemical changes in $A D$. To this end, we investigated molecular changes downstream of the glutamate/GABA neurotransmitters by integrating proteomics measurements from 262 matching DLPFC samples.

Our study provides strong evidence for the metabolic alteration of bioenergetic pathways, cholesterol metabolism, neuroinflammation, osmoregulation, neurotransmission, and other pathways in $A D$ pathogenesis. Further, to maximize utilization of our study by the scientific community, we have made both our data and our findings available through the AD Knowledge Portal and an interactive web resource at https://omicscience.org/apps/brainmwas/. 


\section{Results}

\subsection{Cohort description and characteristics of brain metabolomics data}

We analyzed brain samples of 500 participants from the Religious Order Study and the Rush Memory and Aging Project (ROS/MAP) cohorts ${ }^{11,12}$, including 352 females and 148 males, with a mean age at death of 91 (Table 1). Following enrollment in the study, participants were evaluated for physiological and cognitive function once per year (Figure 1). Neuropathology was assessed after autopsy. Out of the 500 participants, 220 were diagnosed with AD (with or without a secondary cause of dementia) at the time of death, 119 had mild cognitive impairment, 153 were without cognitive impairment, and 8 had other forms of dementia. Samples from the dorsolateral prefrontal cortex (DLPFC) brain region were used for untargeted metabolic profiling. Metabolomics measurements were analyzed in relation to eight AD-related traits covering late life cognitive assessments and postmortem pathology: Clinical diagnosis at the time of death, level of cognition proximate to death, cognitive decline during lifetime, $\beta$-amyloid load, tau tangle load, global burden of AD pathology (global NP), NIA-Reagan score, neuropathological diagnosis inferred based on combination of Braak stage and CERAD score (NP diagnosis, see methods for diagnostic criteria). A detailed description of these AD-related traits is provided in Supplementary Table 1.

The metabolomics platform identified 667 metabolites from various chemical classes (superpathways) in the brain samples, including lipids (42.7\%), amino acids $(22.6 \%)$, nucleotides $(6.7 \%)$, carbohydrates $(6.3 \%)$, cofactor and vitamins $(4.3 \%)$, xenobiotics $(3.7 \%)$, peptides $(2.1 \%)$, and energy-related metabolites (1.5\%) (Figure 2a, Supplementary Table 2). Previous bloodbased metabolomics studies reported strong influences of medications and supplements (such as vitamins) on metabolic profiles ${ }^{4}$. To investigate such effects in brain tissue-based metabolic profiles, we examined influences of 103 grouped medication classes and supplements on metabolic abundances. 552 out of $667(82.75 \%)$ of the metabolites correlated with one or more medications or supplements taken during lifetime. The group of medications to treat benign prostatic hypertrophy associated with the highest number of metabolites (81 metabolites), followed by diuretics (55 metabolites) and multivitamins (52 metabolites). A comprehensive list of medication classes and their effect on the metabolome is provided in Supplementary Table 3. Given these strong associations with the metabolome, medication effects excluding $A D$ and neurologic drugs were regressed out from the metabolic profiles for all following analyses. Moreover, since the postmortem interval (PMI) before sample collection at autopsy may also impact analyte levels, we investigated its effects and found that 307 metabolites associated with PMI (Supplementary Table 4). PMI was therefore included as a covariate in all following analyses. 
To obtain a baseline understanding of metabolism in brain, we used cognitively normal samples to compute associations between measured metabolites and demographic parameters independent of $A D$. This included metabolic associations with age, BMI, sex, and years of education (as a proxy for socioeconomic status). Two metabolites, 1-methyl-5-imidazoleacetate and N6-carboxymethyllysine, were significantly associated with age at $5 \%$ false discovery rate (FDR). Surprisingly, there were no significant associations with sex, BMI, or education, which is in stark contrast to findings in blood ${ }^{13-15}$. Details of this baseline analysis can be found in Supplementary Table 5.

\begin{tabular}{|c|c|}
\hline Total samples & $N=500$ \\
\hline \multicolumn{2}{|l|}{ Sex } \\
\hline Female & $352(70.4 \%)$ \\
\hline Male & $148(29.6 \%)$ \\
\hline Postmortem interval (hours) & $6.6(5.2,8.7)$ \\
\hline BMI & $25.2(22.5,28.2)$ \\
\hline Years of education & $16.00(13.00,18.00)$ \\
\hline Age at death & $91(87,95)$ \\
\hline \multicolumn{2}{|l|}{ APOE4 alleles } \\
\hline 0 & $374(75 \%)$ \\
\hline 1 & $121(24 \%)$ \\
\hline 2 & $5(1 \%)$ \\
\hline \multicolumn{2}{|l|}{ Clinical diagnosis at death } \\
\hline$A D$ & $220(44 \%)$ \\
\hline Mild cognitive impairment (MCI) & $119(23.8 \%)$ \\
\hline No cognitive impairment ( $\mathrm{NCl})$ & $153(30.6 \%)$ \\
\hline Other & $8(1.6 \%)$ \\
\hline & Format: $\mathrm{N}(\%)$ or median (IQR) \\
\hline
\end{tabular}

Table 1. ROS/MAP cohort overview. Postmortem interval refers to the time between death and sample preservation. $\mathrm{BMI}=$ body mass index. IQR = interquartile range, i.e., middle $50 \%$ of the data. 


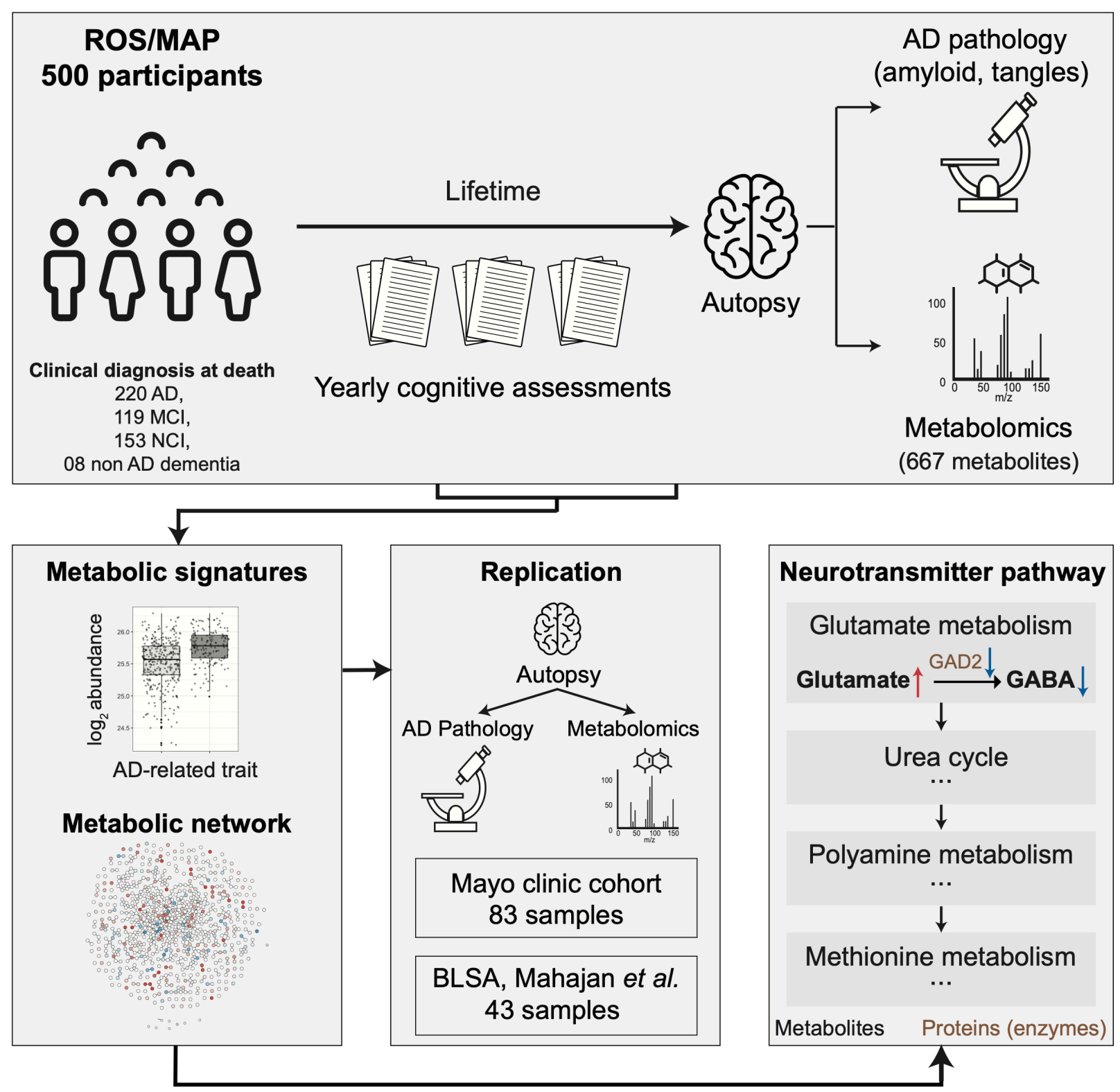

Figure 1: Study overview. 500 ROS/MAP participants were included in this analysis. For each participant, data was available on cognitive assessments during lifetime, postmortem AD brain pathology, and brain metabolic profiles from the dorsolateral prefrontal cortex (DLPFC) region. Metabolic profiles were investigated for associations with AD-related traits and a metabolite interaction network was inferred using a Gaussian graphical model (GGM). Associations were tested for replication in 83 temporal cortex samples from the Mayo Clinic brain bank cohort and compared to a previously published brain-based study. Finally, various pathways previously implicated in AD were metabolically characterized, and a detailed metabolomic/proteomic characterization of the glutamate/GABA neurotransmitter pathway was generated. 


\section{2 $A D$ is associated with widespread metabolic alterations in brain}

To assess AD-related metabolic changes, we computed statistical associations between metabolic profiles and the 8 AD-related traits. All statistical models accounted for AD-related confounders (age, sex, years of education, BMI, and copies of APOE4) as well as postmortem interval. A total of 298 out of 667 metabolites (44.7\%) were significantly associated with one or more $A D$ traits at $5 \%$ false discovery rate (FDR). 80 out of the 298 metabolites showed unique associations with just one of the traits. A total of 218 metabolites associated with more than one trait, which is likely due to high correlations across traits (Figure 2b, Supplementary Figure 1). The majority of the 298 metabolites was associated with one of three AD traits: Cognitive decline ( $n=201)$, tau tangles ( $n=188$ ), and global burden of pathology $(n=183$ ) (Figure $2 c)$. Interestingly, only 34 metabolites associated with $\beta$-amyloid, which was the lowest number of associations among the eight AD traits. Furthermore, we observed that 159 out of the 298 metabolites $(53.4 \%)$ were associated with both premortem parameters and postmortem pathological assessments. All statistical results are provided in Supplementary Table 6. Sexbased stratified analysis revealed that 29 of the 298 metabolites $(10 \%)$ showed associations with at least one trait that were significantly modulated by sex (Supplementary Table 7), and APOE4stratified analysis showed that associations of 77 metabolites (26\%) were influenced by APOE4 status (Supplementary Table 8).

To illustrate the strength of the observed associations, we provide two of the most significant associations in the dataset as examples: Glycerophosphoethanolamine (GPE) levels positively associate with cognitive decline (FDR: 7.05e-13, Figure 2d, left) and N-acetylglutamate negatively associate with global AD pathology (FDR: 3.59e-08, Figure 2d, right). GPE levels were higher with lower cognitive abilities, which corroborates previously published findings ${ }^{16} . \mathrm{N}-$ acetylglutamate levels showed lower levels with higher AD pathology load.

The 298 metabolites that associated with AD-related traits were distributed across all superpathways, including 113 (37.92\%) within the largest super-pathway of lipids, followed by amino acids with $78(26.17 \%)$ associations, and the rest in remaining six super-pathways (Figure 2e). At the more fine-grained sub-pathway level, metabolic associations were distributed across 72 out of the 101 sub-pathways covered in the data (Supplementary Figure 2).

We statistically inferred a metabolic network and annotated it with effect directions and the lowest adjusted $p$-value across the eight $A D$ traits (Figure 2f). The network is based on a Gaussian graphical model (GGM), which corresponds to a data-driven representation of biochemical pathways ${ }^{17,18}$. GGMs have previously been used to systematically investigate various trait effects on the metabolome ${ }^{13,19}$. To further explore our findings networks for each AD trait are available as a Cytoscape file (Supplementary File 1), as well as an interactive online version at https://omicscience.org/apps/brainmwas/. 
Taken together, this analysis revealed global metabolic changes with respect to various ADrelated clinical and neuropathological traits. These alterations encompass all measured metabolic super-pathways, highlighting the massive impact of the disease on brain metabolism.
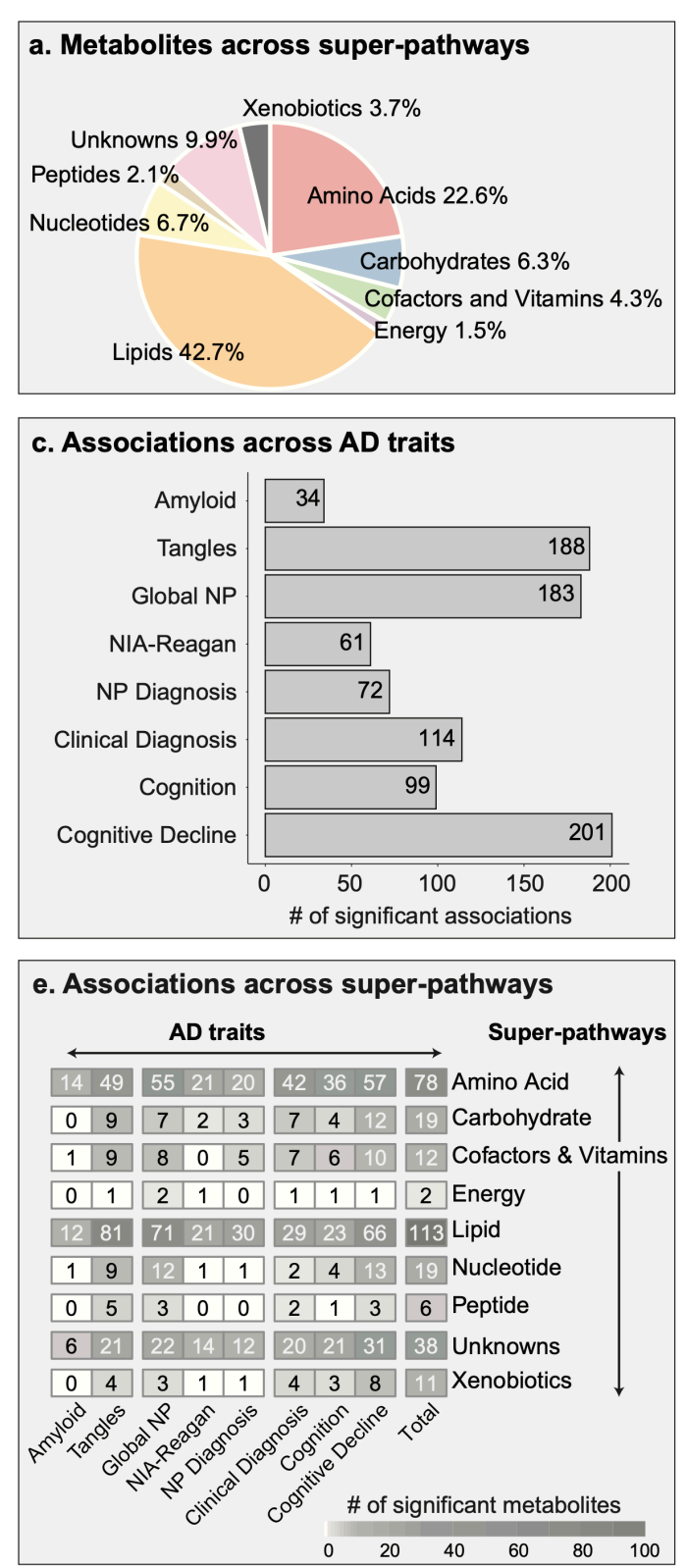
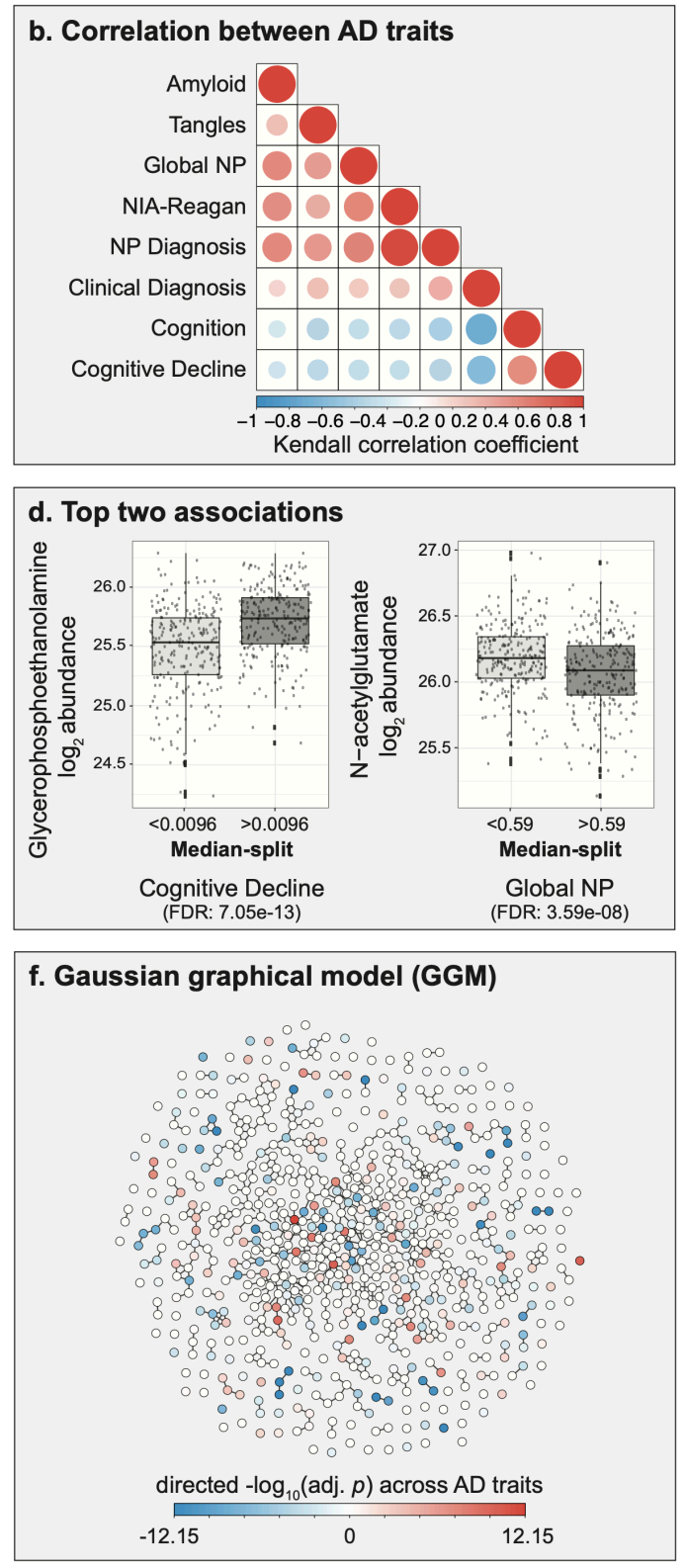

Figure 2: Overview of metabolic associations with AD. a, Metabolites measured in brain samples are distributed across various metabolic classes, referred to as "super-pathways" throughout the manuscript. b, Kendall correlations across the eight AD-related traits. c, A total of 298 metabolites were associated with at least one of the eight AD-related traits. d, Examples of two metabolites with the lowest adjusted p-values. Note that the traits were discretized (mediansplit) for visualization. e, Distribution of metabolic associations across super-pathways. f, Gaussian graphical model of metabolites. Metabolites are colored based on the negative $\log _{10}$ of the lowest adjusted $p$-value across AD-related traits multiplied with the direction of the respective effect estimate. Global NP = Global burden of AD neuropathology. NP Diagnosis $=$ postmortem diagnosis based on Braak stage and CERAD score. 


\subsection{Conditional analysis suggests tau pathology as driver of metabolic changes in brain}

According to the $\beta$-amyloid hypothesis of $A D, \beta$-amyloid is key to $A D$ pathogenesis ${ }^{20}$. It is considered to influence the accumulation of tangles of phosphorylated tau as well as tangle-driven pathogenesis ${ }^{21}$. As a result, $\beta$-amyloid has been the focus of most therapeutic approaches ${ }^{22-26}$. However, recent evidence suggests that tau tangles might be acting independent of $\beta$-amyloid ${ }^{27}$. To identify metabolic signatures specific to $\beta$-amyloid and tau tangles we performed conditional analyses by adjusting for the respective other neuropathology (Figure 3a). In our standard association analysis, i.e., without accounting for $\beta$-amyloid load, 188 metabolites were associated with tau tangle load. 119 out of these 188 associations were still significant after accounting for $\beta$-amyloid load in the statistical model. While 34 metabolites associated with $\beta$-amyloid load in the standard association analysis, only one remained significant after accounting for tau tangle load. Details of the standard and conditional analysis are available in Supplementary Table 6 and Supplementary Table 9, respectively. Taken together, this analysis suggests that metabolic associations of tau tangles are largely independent of $\beta$-amyloid load, while metabolic associations of $\beta$-amyloid load are confounded by tau tangle load.

To corroborate this finding with another omics layer, we performed the same analysis on proteomics profiles. In the standard association analysis, 695 proteins associated with tau tangle load, i.e., without accounting for $\beta$-amyloid load. 252 out of these 695 were still associated with tau tangle load after accounting for $\beta$-amyloid load in our statistical model. While 265 proteins were associated with $\beta$-amyloid load in the standard association analysis, only 68 of these remained correlated after accounting for tau tangle load (Figure 3b). Details of the proteomics standard and conditional analysis are available in Supplementary Table 10.

Taken together, metabolic associations were more widespread for tau tangles and less dependent on $\beta$-amyloid load, which was partially confirmed by a similar trend in the proteomics data. 
bioRxiv preprint doi: https://doi.org/10.1101/2021.11.15.468698; this version posted November 19, 2021. The copyright holder for this preprint (which was not certified by peer review) is the author/funder, who has granted bioRxiv a license to display the preprint in perpetuity. It is made available under aCC-BY-NC-ND 4.0 International license.

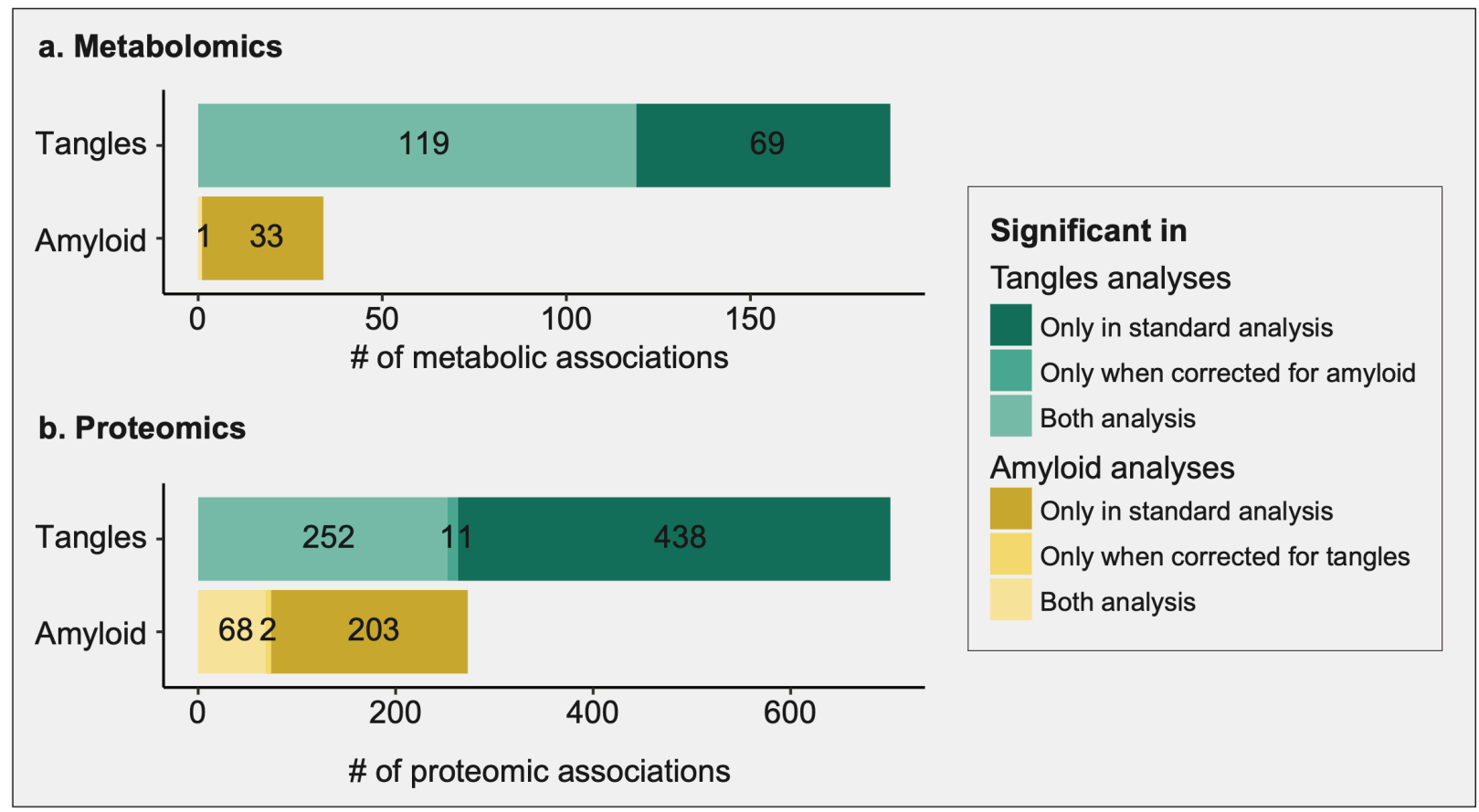

Figure 3: Comparison of standard and conditional analyses of $\beta$-amyloid and tau tangles. Tangle-associated signals appeared largely independent of $\beta$-amyloid signals, while $\beta$-amyloid signals were more strongly dependent on tau tangle signals. $\mathbf{a}, \mathbf{b}$ Overlap of tangle- and $\beta$-amyloid-associated metabolites and proteins, respectively, with and without adjusting for the respective other neuropathology. 


\subsection{AD-associated metabolic alterations overlap across independent brain studies}

To strengthen confidence in our findings, we performed replication analysis using a metabolomics dataset from a Mayo Clinic brain bank cohort. In addition, we compared our results to a previously published brain metabolomics study based on samples from the Baltimore Longitudinal Study of Aging (BLSA) $)^{5}$. Detailed replication results of Mayo can be found in Supplementary Table 11 and published BLSA results used for comparison can be found in Supplementary Table 12.

In the Mayo data, 83 temporal cortex brain samples were used for untargeted metabolic profiling, including $63 \mathrm{AD}$ patients and 20 controls. Of the 8 AD-related traits used in the discovery phase with ROS/MAP cohort, neuropathology-based diagnosis was the only matching trait available in this cohort. Individual measures of neuropathology were not comparable between cohorts, and cognitive assessments were not available for the Mayo cohort. A total of 257 metabolites of the 298 significant in ROS/MAP cohort were measured in the Mayo cohort. 30 of these 257 metabolites were associated with $A D$ in both datasets, i.e., with $A D$ diagnosis in the Mayo cohort and with at least one of the eight AD-related traits in ROS/MAP (Figure 4a), all of which showed consistent effect directions.

In the BLSA study ${ }^{5}, 43$ samples from the inferior temporal gyrus (ITG) and middle frontal gyrus (MFG) brain regions were used for targeted metabolic profiling. The study identified 130 metabolites, of which the authors focused on 26 , which were further categorized into different biochemical groups. In their analysis, 9 out of 26 metabolites associated with AD diagnosis. All 26 metabolites were measured in our study, 17 of these 26 associated with AD-related traits, and 6 of these 17 were among the 9 metabolites associated in their study (Figure 4b). Of those 6 metabolites, 5 had the same effect directions, while cysteine was found to be upregulated in the BLSA study and downregulated in ROS/MAP.

Overall, 35 of the 298 associations identified in ROS/MAP were confirmed with consistent effect directions in either the Mayo or the BLSA cohort. 
bioRxiv preprint doi: https://doi.org/10 1101/2021.11.15.468698; this version posted November 19, 2021. The copyright holder for this preprint (which was not certified by peer review) is the author/funder, who has granted bioRxiv a license to display the preprint in perpetuity. It is made available under aCC-BY-NC-ND 4.0 International license.

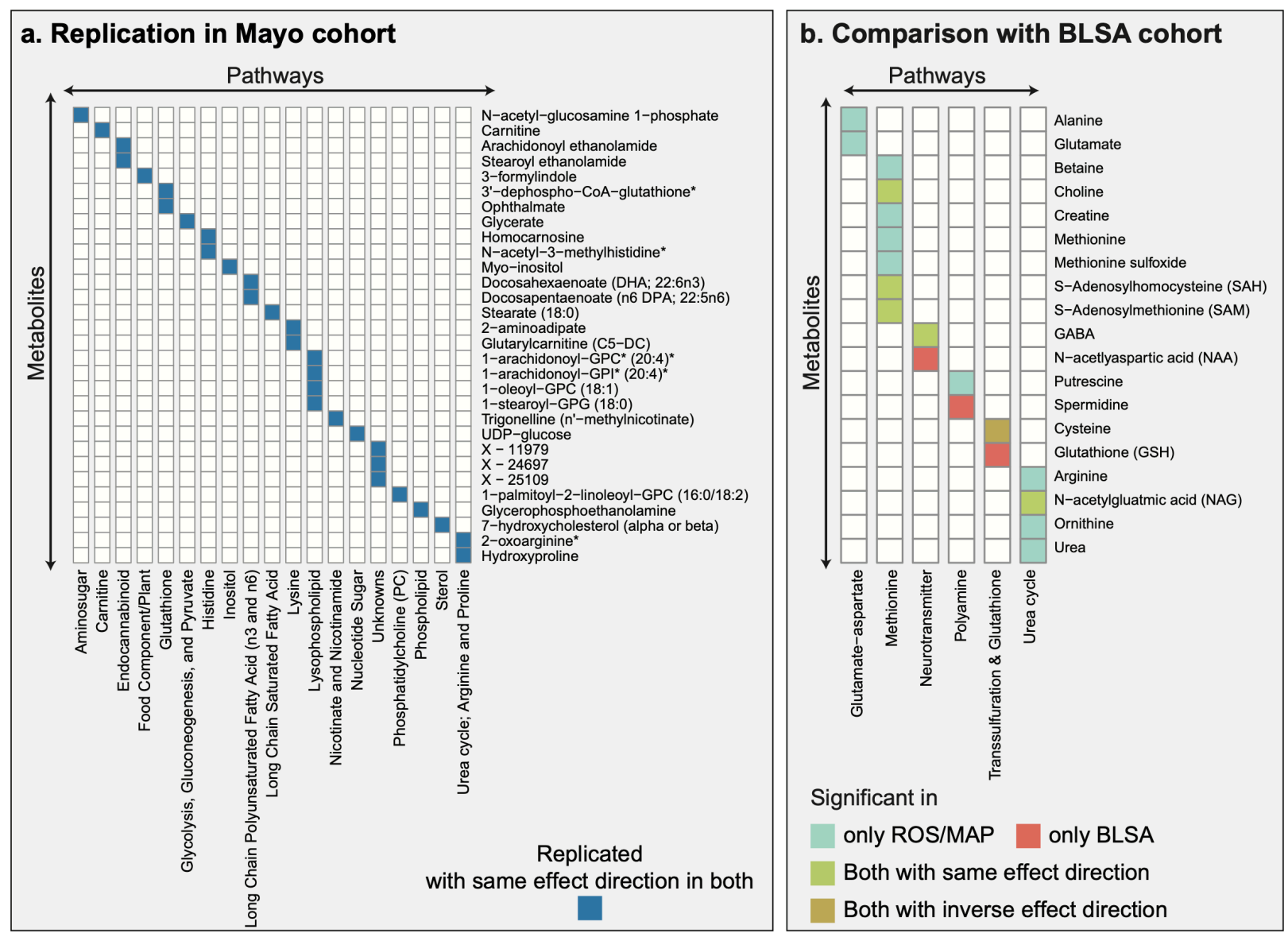

Figure 4: Overlap across independent cohorts and brain-regions. a, The Mayo and ROS/MAP cohorts have 30 metabolic associations with consistent effect directions in common. b, The BLSA and ROS/MAP cohorts have 5 metabolic associations with consistent effect direction in common (green and brown). Cysteine showed inconclusive effect directions, with a positive association with AD in the BLSA cohort and a negative association with AD in the ROS/MAP cohort. 


\subsection{Metabolic alterations further characterize pathways previously implicated in AD}

To examine the contribution of metabolic alterations in previously reported AD-related pathogenic processes, we selected four pathway groups for further exploration (Figure 5). Comprehensive functional annotations based on Metabolon's sub-pathways are available in Supplementary Figure 2. For each pathway group discussed below, we included metabolites associated with at least one of the eight $A D$-related traits.

Bioenergetic pathways: Bioenergetic dysregulation is a hallmark of $A D$, which has been demonstrated using different technologies, from PET neuroimaging to deep molecular profiling such as metabolomics and proteomics studies ${ }^{28-31}$. In our analysis, key metabolites from bioenergetic pathways, including glycolysis, branched-chain amino acid (BCAA) metabolism, and mitochondrial $\beta$-oxidation, were found to be deregulated in AD. This included positive correlations of the glycolytic metabolites glucose, glycerate, glucose 6-phosphate, and 1,5-anhydroglucitol; the BCAAs valine, isoleucine, and leucine, as well as their 1-carboxyethyl conjugates and degradation products $\beta$-hydroxyisovalerate, and 3-hydroxyisobutyrate; and the acylcarnitines isobutyrylcarnitine (C4), tiglyl carnitine (C5), 2-methylbutyrylcarnitine (C5), glutarylcarnitine (C5-DC), and 5-dodecenoylcarnitine (C12:1). High abundances of these metabolites resemble observations in blood metabolic profiles of individuals with type 2 diabetes and stand in contrast to blood-based studies in $A D$, which reported negative associations of, e.g., BCAA levels with $A D^{4,32}$. Together, these results are in line with the hypothesis that AD might represent a "type 3" diabetes that selectively affects the brain ${ }^{33}$.

Cholesterol metabolism and sterol pathway: The strongest genetic risk for $A D$ is exerted by variants of the APOE gene, a lipoprotein involved in cholesterol transport and metabolism ${ }^{34-36}$. Our findings provide further evidence for the AD-associated significance of this pathway, with degradation products of cholesterol showing positive correlations with AD-related traits. These degradation products include 7a-hydroxy-3-oxo-4-cholestenoic acid (7-HOCA), which has been described as a CSF-based marker for blood-brain-barrier integrity ${ }^{37}$; 4-cholesten-3-one, a product of cholesterol oxidation and isomerization through bacterial enzymes ${ }^{38}$; and 7-hydroxycholesterol, a precursor for bile acids. Notably, cholesterol itself did not show any significant associations, indicating potential dysfunctional cholesterol clearance rather than a direct role of cholesterol in $A D$. This hypothesis is further supported by previous studies where we observed a significant increase of secondary bile acids in $A D^{7,33,39}$. 
Neuroinflammation and oxidative stress: Neuroinflammation is a central pathogenic feature of $A D$ and is accompanied by the production of reactive oxygen species leading to oxidative stress ${ }^{40}$. $A D$ has been associated with both lipid mediators of inflammatory processes as well as immune response, including eicosanoids, and molecules involved in the antioxidant defense, such as glutathione ${ }^{41-43}$. In line with these findings, we observed significant positive correlations of metabolites in the glutathione pathway with $A D$, indicating an upregulated antioxidant response. Significant metabolites included 4-hydroxy-nonenal-glutathione, a marker for detoxification of lipid peroxidation through glutathione S-transferases (GSTs) ${ }^{44}$; cysteinylglycine disulfide, a degradation product of oxidized glutathione ${ }^{42}$; and ophthalmate, an endogenous analog of hepatic glutathione $(\mathrm{GSH})$ and potential marker for $\mathrm{GSH}$ depletion ${ }^{45}$. Moreover, pro-inflammatory eicosanoids showed positive associations with AD, including 15-oxoeicosatetraenoic acid (15-KETE), which has been linked to GST inhibition ${ }^{46}$, and 12-hydroxy-heptadecatrienoic acid (12-HHTrE), overall providing further molecular evidence for active inflammatory processes in AD. In contrast, anti-inflammatory long-chain omega-3 polyunsaturated fatty acids (PUFAs), such as eicosapentaenoate (EPA) and docosahexaenoate $(\mathrm{DHA})^{47,48}$, were negatively associated with AD.

Osmoregulation. Osmolytes are a class of molecules that primarily sustain cell integrity ${ }^{49}$. They have been suggested to play a neuroprotective role in AD by activating mTOR-independent autophagy signaling to inhibit accumulation of $\beta$-amyloid plaques ${ }^{50}$. Osmolytes also affect protein folding ${ }^{51}$, and their therapeutic potential has been discussed in $A D$ as well as other neurodegenerative proteinopathies ${ }^{52}$. Moreover, osmolyte imbalances can impact neuronal hyperexcitation by influencing neurotransmitter uptake ${ }^{49}$. In our analysis, we observed positive associations of several osmolytes with $A D$, including 2-aminoadipate, arginine, glycerophosphorylcholine (GPC), myo-inositol, serine, and urea, whereas betaine was negatively associated with the disease. As these observations are based on bulk tissue metabolomics, it remains unclear if these metabolites are deregulated within or outside of the cell. Nevertheless, the strong statistical significance underlying these associations suggest an important role of osmoregulation in AD which warrants further investigation. 


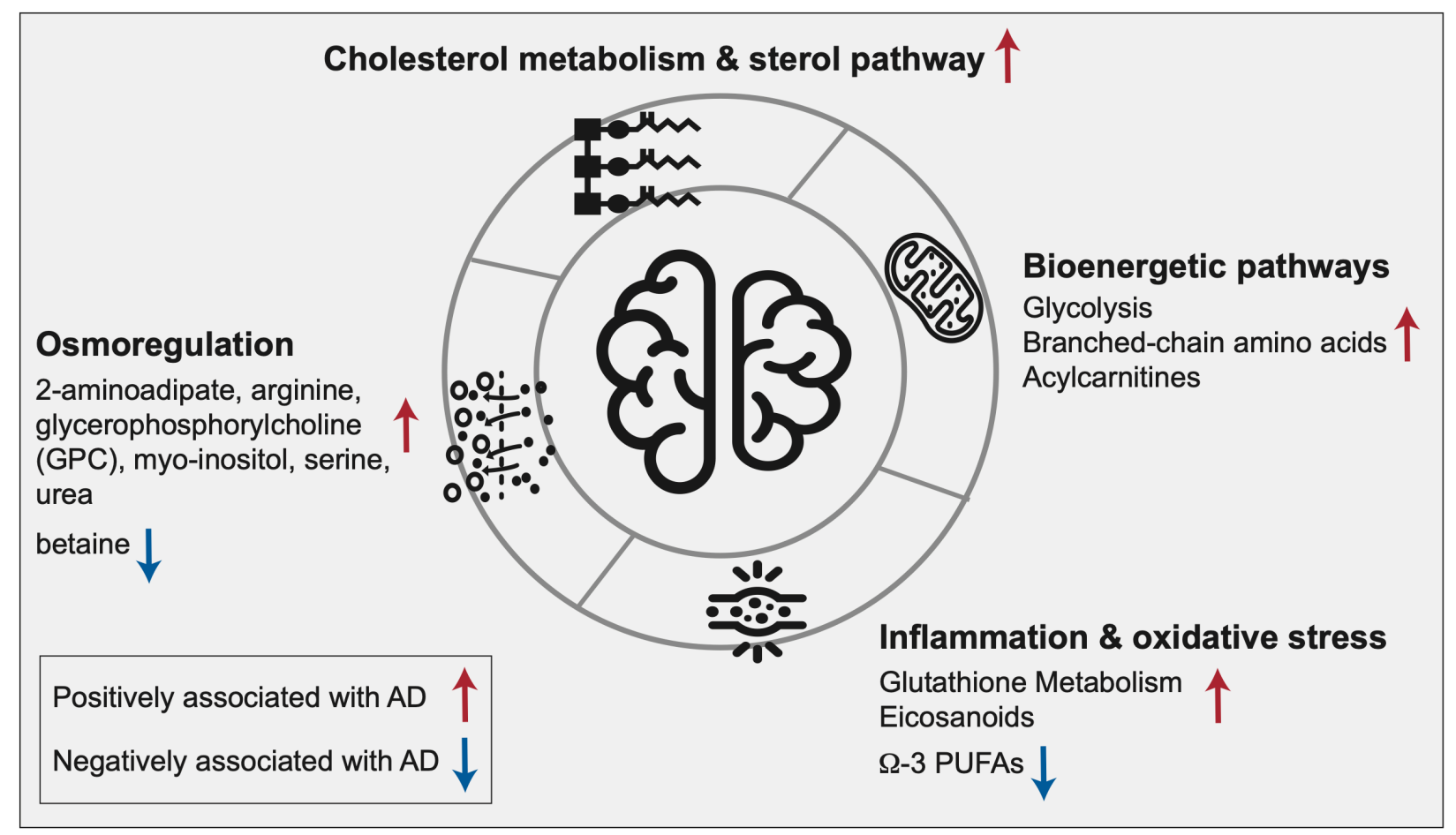

Figure 5: Pathway-level metabolic associations with AD-related traits. The highlighted biological processes have previously been implicated in AD. Our data provides a metabolic characterization of the alterations of these pathways in the AD brain: Cholesterol metabolism has an established connection to late-onset AD through APOE4, the major genetic risk factor for the disease. Bioenergetic dysregulation is one of the earliest detectable changes in the central nervous system in $A D$ and has also been described in the periphery. Inflammation and oxidative stress have been reported to synergistically affect $A D$ pathogenesis. Osmoregulation affects various aspects of $A D$ pathology, including protein folding, neural excitation, and autophagy. 


\subsection{Integration of complementary omics provides comprehensive view of biochemical cascade downstream of neurotransmitters}

As a detailed showcase of the complex, biochemical interconnections in brain omics data, we selected a biochemical cascade downstream of the neurotransmitters glutamate and gamma aminobutyric acid (GABA). An elevated synaptic excitatory/inhibitory (E/l) ratio of these neurotransmitters has been linked to hyperexcitability and cognitive impairment observed in $A D^{53,54}$. Furthermore, given GABA's positive correlation with efficient working memory within the DLPFC region $^{55}$, it is of high significance to investigate GABA-related deregulation in this region.

We compiled biochemical steps of metabolites and enzymes downstream of glutamate using known reactions from the public database pathbank ${ }^{56}$ (Figure 6). Notably, the cascade does not contain the routes from GABA to glutamate or from putrescine to GABA due to a lack of coverage of metabolites along those pathways.

Based on proteomics profiles available for 262 matching brain samples, we performed a targeted association analysis of AD-related traits and proteins that are enzymatically involved in this pathway cascade (Supplementary Table 13). Significant metabolic and proteomic associations with at least one of the eight AD-related traits were annotated on the respective molecules within the cascade.

The pathway cascade starts with glutamate, which was positively associated with AD in our data. Excitatory glutamatergic synapses involving $\mathrm{N}$-methyl D-aspartate receptors (NMDAR) have previously been targeted by memantine to treat severe $A D^{57}$. Glutamate is the precursor of the inhibitory neurotransmitter GABA, which we found to be negatively associated with AD. Interestingly, protein abundance of glutamate decarboxylase (GAD2), which catalyzes the production of GABA from glutamate was also negatively associated with AD pathology in our data. This negative association provides a potential explanation for the imbalance between the two neurotransmitters. Glutamate metabolism is directly connected to the urea cycle, in which ornithine, arginine, and urea were positively associated with AD. Urea buildup to neurotoxic levels has been observed in postmortem brains of Huntington's disease and has furthermore been linked to dementia ${ }^{58}$. Inhibition of arginase (ARG2) has been suggested to reduce the production of urea ${ }^{59}$. Arginase was positively associated with AD in our data; it catalyzes the conversion of arginine to ornithine, with urea as a byproduct. Urea cycle further feeds into the polyamine pathway, in which putrescine was negatively associated with $A D$, while spermidine and spermine were not significantly associated with AD. Putrescine promotes the clearance of apoptotic cells via efferocytosis ${ }^{60}$, a mechanism affected in $A D$ and other neurodegenerative diseases ${ }^{61}$. The enzyme S-methyl-5'-thioadenosine phosphorylase (MTAP) links the polyamine pathway to methionine metabolism, in which methionine, methionine sulfoxide, s-adenosylmethionine, and s-adenosylhomocysteine were positively associated with $A D$, with concordant changes in protein levels of respective enzymes MTAP, mitochondrial peptide methionine sulfoxide reductase (MSRA) and methionine adenosyltransferase (MAT2A). In a previous study, we have shown that 
bioRxiv preprint doi: https://doi.org/10.1101/2021.11.15.468698; this version posted November 19,2021 . The copyright holder for this preprint

(which was not certified by peer review) is the author/funder, who has granted bioRxiv a license to display the preprint in perpetuity. It is made available under aCC-BY-NC-ND 4.0 International license.

higher levels of methionine in CSF were associated with $\mathrm{AD}^{8}$. Methionine acts as an antioxidant by forming methionine sulfoxide and is a precursor of s-adenosylmethionine, which is a key methyl donor in brain cells and involved in the synthesis of the neurotransmitters dopamine, epinephrin, and serotonin via the folate cycle ${ }^{62}$.

Overall, our analysis provides an integrated, multi-omics view of neurotransmitter-related changes known to play a role in the pathogenesis of AD. 


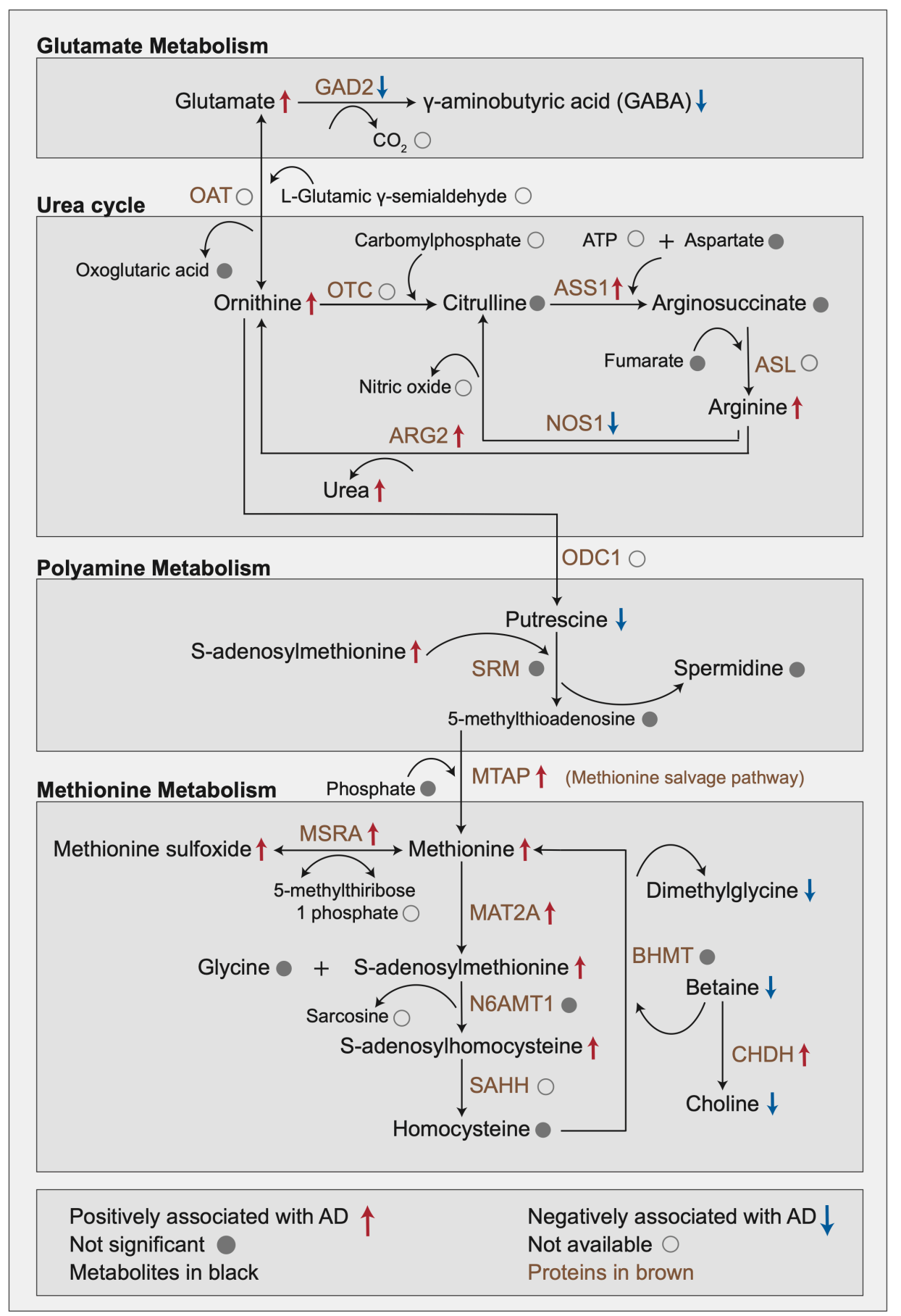

Figure 6: Metabolic changes downstream of the neurotransmitters glutamate/GABA. This multi-omics cascade starts with biochemical process involving conversion of glutamate into GABA within glutamate metabolism. Glutamate metabolism feeds into the urea cycle by conversion of glutamate to ornithine. Urea buildup to neurotoxic levels has been observed in postmortem brains of Huntington's disease and has furthermore been linked to dementia. Urea cycle connects to polyamine metabolism via conversion of ornithine into putrescine. Putrescine promotes the clearance of apoptotic cells via efferocytosis, a mechanism affected in AD. Polyamine metabolism connects to methionine metabolism though methionine salvage pathway. Methionine acts as an antioxidant and is a precursor of sadenosylmethionine, which is a key methyl donor in brain cells and involved in the synthesis of neurotransmitters dopamine, epinephrin, and serotonin via folate cycle. 


\section{Discussion}

In this work, we provide a global view of metabolic changes in brain related to Alzheimer's disease. Our study is based on broad untargeted metabolomics profiles of 500 brain tissue samples from the DLPFC, covering 667 metabolites from various biochemical classes. We demonstrated that in cognitively normal individuals, age, sex, education, and BMI did not show major effects on brain metabolites. These limited associations of brain metabolites with demographics and socioeconomic status stand in contrast to the strong associations seen with blood metabolic profiles ${ }^{13-15}$. Conversely, intake of medications had major effects on brain metabolome, as observed in blood metabolic profiles ${ }^{4}$, highlighting the importance to account for effects of pharmaceuticals.

In the subsequent association analysis, we found that 298 out of the 667 metabolites correlated with at least one of the eight investigated AD-related traits, covering cognition and several neuropathological parameters. We confirmed 30 of our associations using independent samples from the Mayo Clinic brain bank cohort. Additionally, 5 associations were confirmed using a study on the BLSA cohort ${ }^{5}$. Two pathways, urea cycle and glutathione metabolism, were associated with $A D$ in all three cohorts. This overlap was observed despite the substantial differences in sample sizes, profiled brain regions, study designs, and clinical parameters. We thus conclude that the 35 metabolites and two pathways are high confidence AD-related metabolic signals in brain tissue, and the metabolic associations unique to our ROS/MAP study need further validation. Of note, we observed significant modulation of metabolic associations through sex and APOE4 status, which is concordant with previous findings in blood-based metabolomics data ${ }^{3}$.

We explored our findings in the context of various functional processes that have been previously implicated in $A D$, including bioenergetic pathways, cholesterol metabolism, neuroinflammation, and osmoregulation. Our study extends the view on these AD-related pathways through metabolic alterations in brain. Of these processes, metabolic alterations of osmoregulation within the central nervous system have, to the best of our knowledge, so far not been studied in detail. Osmolytes participate in multiple critical processes associated with neurogenerative diseases including protein folding ${ }^{63}$, autophagy ${ }^{50}$, and hyperexcitation of neurons ${ }^{49}$. While our observations might to some extent be confounded by, e.g., systematic differences in the hydration status of AD patients prior to death, the large number and strong significance of associations within this class suggests a potentially functional link to pathomechanisms in AD. 
Moreover, we investigated detailed biochemical alterations downstream of glutamate and GABA. Imbalances of these neurotransmitters have previously been associated with hyperexcitability and cognitive impairment in $A D^{53,54}$. In our study, the excitatory neurotransmitter glutamate was positively associated with $A D$, while the inhibitory neurotransmitter GABA was negatively associated with $A D$. To investigate the downstream effects of this excitatory/inhibitory imbalance, we explored the metabolic and enzymatic changes in the biochemical cascade starting from the conversion of glutamate to GABA, connecting glutamate to urea cycle, polyamine metabolism, and methionine metabolism. Our study shows how the integration of metabolomics with proteomics provides a comprehensive overview of biochemical changes downstream of these neurotransmitters. Moreover, to the best of our knowledge, this is the first reporting of low levels of GABA in $A D$ within the DLPFC region. DLPFC is associated with working memory in individuals ${ }^{55}$, which becomes impaired during $A D$ pathogenesis ${ }^{64}$. Thus, GABA levels within the DLPFC region have been of considerable interest to the $A D$ community ${ }^{64}$, which is corroborated by our results.

Addressing the complex interplay of $\beta$-amyloid deposition and tau tangles in $A D$, we performed a conditional statistical analysis. In our data, $97 \%$ of the $\beta$-amyloid-associated metabolites were dependent on tau tangle load, while only $36.7 \%$ of the tangle-associated metabolites were dependent on $\beta$-amyloid load. Our study thus provides preliminary evidence that the metabolic component of tangle-driven pathogenesis is independent of $\beta$-amyloid, which is in line with recent literature that suggests that tau accumulation might be independent of $\beta$-amyloid ${ }^{27}$. This finding may also suggest that metabolic changes in the brain are mostly later events in the pathologic cascade of $A D^{65}$ and closer temporally to tau pathology, neurodegeneration and cognitive decline than to $\beta$-amyloid accumulation. Further supporting this, the largest number of associations in ROS/MAP were detected with cognitive decline, an event deemed to be at the later stages of the pathologic cascade of events in $A D^{66}$.

Despite the many novel insights into metabolic alterations in brain observed in AD, our study has some limitations. First, cross-sectional studies cannot assess the causal direction of the identified associations. That is, an observed metabolic change in AD could be a factor directly contributing to disease development, or it could be a downstream effect of the pathological changes in brain. The true effect direction can only be determined in mechanistic follow-up studies or by genetic causality analysis such as Mendelian randomization ${ }^{67}$, for which our study did not have the necessary statistical power. Second, postmortem tissue samples are prone to substantial biological and technical variation, as seen in the association of 307 out of 667 metabolites with postmortem interval $(\mathrm{PMI})$, i.e., the time between death and sample preservation. Despite the statistical correction for PMI interval, degradation of certain metabolites until sample preservation is a factor that cannot be controlled in this type of study. 
Follow-up studies will be needed to build upon our findings, to complete the picture of dysregulated metabolism and pathological pathways in the Alzheimer's disease brain. In particular, the wide availability of multi-omics datasets will provide a more holistic picture of the molecular changes associated with the disease ${ }^{68-70}$. The integration of proteomics data into the glutamate/GABA pathway exploration in our study represents a pilot analysis in this direction; however, large-scale studies with an "ome-wide" integration of the (epi-)genome, transcriptome, proteome, and metabolome are required to further elucidate the mechanistic basis of $A D$ pathogenesis and outline potential treatment options. To enable these efforts, we published raw and processed metabolomics data through the AD Knowledge Portal provide all analysis codes, as well as the interactive reference catalog of hundreds of associations reported in this study in an accompanying web portal to the research community. 


\section{Methods}

\subsection{Cohorts, clinical data, and neuropathological data}

ROS/MAP cohort: The Religious Order Study (ROS) and Rush Memory and Aging Project (MAP) cohorts ${ }^{11,12}$ are two longitudinal, clinicopathologic studies conducted by the Rush Alzheimer's Disease Center. ROS started in 1994 with the recruitment of individuals from religious communities across the United States. MAP started in 1997 with the recruitment of individuals from a wide range of backgrounds and socio-economic statuses from northeastern Illinois. Both cohorts were approved by an institutional review board of Rush University Medical Center. Both studies focus on older individuals who agreed to longitudinal clinical analysis and brain donation after death. All participants signed an informed consent, an Anatomic Gift Act, and a repository consent to allow their data and biospecimens to be shared. Following enrollment in the study, participants were evaluated for physical and cognitive function annually. After death, pathologic assessment was performed. 514 samples from DLPFC brain region were used for metabolomics profiling, along with associated metadata, including medications taken during lifetime, age at death, sex, BMI, postmortem interval, APOE genotype status, education history, cognitive scores during lifetime, cognitive decline (computed-based on longitudinal cognitive scores), clinical diagnosis at death, $\beta$-amyloid and tau protein load in brain tissue, global burden of $A D$ neuropathology (mean of neuritic plaques, diffuse plaques, and neurofibrillary tangles), NIAReagan score, Braak stage and CERAD score. Neuropathological diagnosis was derived using the following criteria: $A D$ case status was assigned where Braak stage was $\geq 4$ and CERAD score was $\leq 2$; control case status was assigned where Braak stage was $\leq 3$ and CERAD score was $\geq 3$. All clinical parameters have previously been described in detail ${ }^{71}$.

Mayo Clinic cohort: 84 samples from the temporal cortex brain region were obtained from the Mayo Clinic Brain Bank. Details on this cohort have been provided in previous studies ${ }^{72,73}$. All samples received diagnoses at autopsy following neuropathologic evaluation. Briefly, 64 samples had neuropathologic diagnosis of $A D$ with Braak $\geq 4.0$ and 20 control samples had Braak $\leq 3$ and without any neurodegenerative diagnoses. All 84 samples were from North Americans of European descent with ages at death $\geq 60$ for $A D$ and $\geq 53$ for controls. 
Cohort differences: Three cohorts were used in this publication - ROS/MAP for discovery, Mayo brain clinical cohort for replication and a published Baltimore Longitudinal Study of Aging (BLSA) based study ${ }^{5}$ for comparison. These cohorts have fundamental differences: (a) Participant recruitment. BLSA is an aging study, Mayo Clinic samples are from an archival brain bank with neuropathologic diagnoses of $A D$ and control, while ROS/MAP recruited older people. (b) ADrelated traits. Mayo has diagnosis determined by neuropathology and BLSA has diagnosis determined based on neuropathology and cognitive conditions. ROS/MAP records several neuropathological as well as cognitive scores. (c) Unlike the other two cohorts, ROS/MAP collects various lifetime variables longitudinally, including cognitive scores, lifestyle, medications taken by participants. (d) Sample sizes were lower in BLSA (43), and Mayo (84), compared to ROS/MAP (514). (e) Different brain regions were profiled. BLSA sampled frontal and temporal gyrus, Mayo the superior temporal gyrus of the temporal cortex, and ROS/MAP the dorsolateral prefrontal cortex (DLPFC).

\subsection{Metabolomics profiling}

Brain metabolic profiles were measured using the untargeted metabolomics platform from Metabolon Inc. Briefly, tissue samples were divided into four fractions; two for ultra-high performance liquid chromatography-tandem mass spectrometry (UPLC-MS/MS; positive ionization), one for UPLC-MS/MS (negative ionization), and one for a UPLC-MS/MS polar platform (negative ionization). Peaks were quantified using the area-under-the-curve in the spectra. To account for run-day variations, peak abundances were normalized by their respective run-day medians. Compounds were identified using an internal spectral database. A detailed description of all experimental procedures can be found in supplementary information.

\subsection{Data preprocessing}

ROS/MAP and Mayo metabolomics: Metabolites with over 25\% missing values were filtered out, leaving 667 out of an original 1,055 metabolites for ROS/MAP and 664 out of 827 for Mayo. Probabilistic quotient normalization was applied to correct for sample-wise variation ${ }^{74}$, followed by $\log _{2}$ transformation. Remaining missing values were imputed using a k-nearest-neighborbased algorithm ${ }^{19}$. Outlier samples in the data were removed using the local outlier factor method ${ }^{75}$ implemented in the $\mathrm{R}$ package bigutilsr. To account for remaining irregularly high or low single concentrations, values with absolute abundance above $q=$ abs(qnorm $(0.0125 / n)$ ), with $n$ representing the number of samples, were set to missing. This formula finds the cutoff for values with less than $2.5 \%$ two-tailed probability to originate from the same normal distribution as the rest of the measurement values, after applying a Bonferroni-inspired correction factor (division by sample size). These new missing values were then imputed by another round of the k-nearestneighbor algorithm. 
ROS/MAP proteomics: Proteomics data was downloaded from the AMP-AD Knowledge Portal (https://adknowledgeportal.synapse.org), details of proteomic profiling and data processing can be found in the original publication ${ }^{76}$. Briefly, data were $\log _{2}$-transformed and corrected for batch effects using 'median polish' approach. In our analysis, proteins with over $25 \%$ missing values were filtered out and remaining missing values were imputed using a k-nearest-neighbor-based algorithm ${ }^{19}$. Outliers were treated with same approach as the metabolomics data (see above).

Medication correction: For the ROS/MAP cohort, all prescription and over the counter medications were collected at each study visit. To account for influences of these medications on metabolomics and proteomics, a linear stepwise backward selection approach was used ${ }^{4}$. All preprocessing steps were performed using the maplet $\mathrm{R}$ package ${ }^{77}$.

\subsection{Differential analysis of metabolites and proteins}

ROS/MAP: Five outliers identified by the local outlier factor method, six samples with missing medication information, 1 sample with missing $B M I$ and 2 samples with missing APOE genotype status were removed from further analysis. Therefore, after preprocessing, 500 samples were used for metabolic analysis and 262 matching samples were used for proteomic analysis. Metabolite and protein associations were computed using generalized linear models with the traits as response variables and molecule levels as predictors. For statistical analysis, the following transformations were made: Square root of $\beta$-amyloid load and binarized NIA-Reagan score $(0-$ low likelihood of $A D, 1$ - high likelihood of $A D)$. For the association analysis with clinical diagnosis, 8 non-AD related dementia samples were removed. Appropriate link functions were used according to the respective variable types, i.e., identity link function for continuous traits (regular linear regression for $\beta$-amyloid, tau tangles, global burden of pathology, cognition levels, cognitive decline), logit for binary traits (logistic regression for NIA-Reagan score and NP diagnosis), and probit for the ordinal trait (ordinal regression for clinical diagnosis after death). All models accounted for confounding effects of age, sex, BMI, postmortem interval, number of years of education, and number of $A P O E \varepsilon 4$ alleles. Notably, age, sex, years of education did not show much influence on metabolic profiles of cognitively normal samples, but are known confounders of $A D^{3}$, justifying the correction in the models. To account for multiple hypothesis testing, $p$-values were corrected using the Benjamini-Hochberg $(\mathrm{BH})$ method $^{78}$. Cognitive decline and cognition levels are inversely related to $A D$, and thus the direction of association was reversed for those two traits after statistical analysis. 
Mayo: One AD sample with missing APOE genotype status was removed from further analysis. Therefore, after preprocessing, $63 \mathrm{AD}$ and 20 control samples with complete information on age at death, $A P O E \& 4$ allele status, and sex were used for our analysis. For replication, metabolites that associated with any of the eight AD-related traits in ROS/MAP cohort at 5\% FDR were selected. The analysis was performed using two subsequent logistic regressions with diagnosis as outcome. The first model was built without any confounder correction. To account for multiple hypothesis testing, p-values were corrected using the Benjamini-Hochberg $(\mathrm{BH})$ method $^{78}$. Metabolites with adjusted $p$-values $<0.05$ were selected for the second model. The second model was built with confounders sex, number of $A P O E \varepsilon 4$ alleles, and age at death. Metabolites with nominal $p$-values $<0.05$ in the second model were considered replicated. All analyses were performed using the maplet $\mathrm{R}$ package ${ }^{77}$.

\subsection{Stratified analysis}

To determine the influence of sex and $A P O E \varepsilon 4$ status on metabolic associations, we performed a stratified analysis per factor (sex and $A P O E \varepsilon 4$ status) for each AD-related trait. Metabolites significant at 5\% FDR were selected to compute within-group (male/female, APOE $\varepsilon 4^{+} / A P O E \varepsilon 4^{-}$) metabolic associations with AD-related traits. $\beta$ estimates across groups per metabolite were

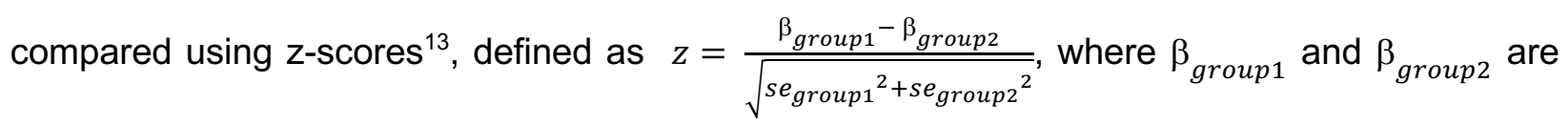
the $\beta$ coefficients from the linear regressions performed in the two groups, and $s e_{\text {group } 1}$ and $s e_{\text {group } 2}$ are the corresponding standard errors. Z-scores are approximately standard normally distributed and were thus used to compute $p$-values using a normal distribution. Any metabolite with a nominal $p$-value $<0.05$ was considered significantly different within the respective group.

\subsection{Metabolic network inference}

To infer the metabolite-metabolite interaction network, a partial correlation-based Gaussian graphical model (GGM) was computed using the GeneNet R package ${ }^{79}$. P-values of partial correlations were corrected using the Bonferroni method. Partial correlations with adjusted p-values < 0.05 were used for network construction between metabolites. To annotate the metabolic network with $A D$ associations, a score was computed for each metabolite/trait combination as follows: $p_{\text {score }}=d *\left(-1 * \log _{10}(p . a d j)\right)$, where $p . a d j$ is the adjusted p-value of the model, and $d$ is direction $(-1 / 1)$ of metabolite association based on test statistic (positive or negative correlation with AD-related trait). To aggregate the signal across the traits, an overall score was defined as the $p_{\text {score }}$ with maximum absolute value. This overall score was used to color the nodes in GGM in Figure $2 f$ and the online supplement. 


\section{Data availability}

The data used in this paper can to be obtained from two sources: (1) Metabolomics data for the ROS/MAP and Mayo cohorts, clinical data for the Mayo cohort, and proteomics data for the ROS/MAP cohort are available via the AD Knowledge Portal (https://adknowledgeportal.org). The $A D$ Knowledge Portal is a platform for accessing data, analyses, and tools generated by the Accelerating Medicines Partnership (AMP-AD) Target Discovery Program and other National Institute on Aging (NIA)-supported programs to enable open-science practices and accelerate translational learning. The data, analyses and tools are shared early in the research cycle without a publication embargo on secondary use. Data is available for general research use according to the following requirements for data access and data attribution (https://adknowledgeportal.org/DataAccess/Instructions). For access to content described in this manuscript see: http://doi.org/10.7303/syn26401311. (2) The full complement of clinical and demographic data for the ROS/MAP cohort are available via the Rush AD Center Resource Sharing Hub and can be requested at https://www.radc.rush.edu.

An interactive network view of $A D$ associations from this study can be found at https://omicscience.org/apps/brainmwas/.

All R scripts to generate the tables and figures of this paper are available at https://github.com/krumsieklab/ad-brain-landscape.

\section{Acknowledgements}

This work was done as part of the National Institute of Aging's Accelerating Medicines Partnership for AD (AMP-AD) and was supported by NIH grants 1U19AG063744, 1R01AG069901-01A1, U01AG061357, P30AG10161, P30AG72975, R01AG15819, R01AG17917, U01AG46152, U01AG61356, RF1AG058942, RF1AG059093, and U01AG061359. The results published here are in whole or in part based on data obtained from the $A D$ Knowledge Portal (https://adknowledgeportal.org).

The Religious Orders and the Rush Memory and Aging studies were supported by the National Institute on Aging grants P30AG10161, R01AG15819, R01AG17917, U01AG46152, and U01AG61356. The NIA also supported the Alzheimer Disease Metabolomics Consortium which is a part of the NIA's national initiatives AMP-AD and M²OVE-AD (R01 AG046171, RF1 AG051550, and 3U01 AG061359-02S1). We thank the participants of ROS and MAP for their essential contributions and the gifts of their brains to these projects. All subjects gave informed consent.

The Mayo Clinic samples are part of the RNAseq study data led by Dr. Nilüfer Ertekin-Taner, Mayo Clinic, Jacksonville, FL as part of the multi-PI U01 AG046139 (MPIs Golde, Ertekin-Taner, Younkin, Price). Samples were provided from the following sources: The Mayo Clinic Brain Bank. 
Data collection was supported through funding by NIA grants P50 AG016574, R01 AG032990, U01 AG046139, R01 AG018023, U01 AG006576, U01 AG006786, R01 AG025711, R01 AG017216, R01 AG003949, NINDS grant R01 NS080820, CurePSP Foundation, and support from Mayo Foundation.

RB thanks her colleagues from Krumsiek lab for fruitful discussions and support in this work.

\section{Author contributions}

RB, MArnold, GK, JK designed the computational and statistical methods, performed the analysis, interpreted the results, and drafted the manuscript. MAW created the interactive online supplement. DAB is PI of the ROS/MAP study and provided samples as well as phenotypic data. NET, XW, MAllen provided samples and phenotypic data on the Mayo clinic cohort and contributed to the analysis of those samples. CB curated and managed phenotypic data. AIL, NTS provided the preprocessed proteomics data for matching ROS/MAP samples and contributed to the analysis of those samples. All authors read and reviewed the manuscript. RK$\mathrm{D}$ acquired funding and is the overall $\mathrm{PI}$ of the Alzheimer's disease metabolomics consortium.

\section{Competing interests}

R.K-D., MArnold, GK are (through their institutions) inventors on key patents in the field of metabolomics including applications for Alzheimer disease. R.K-D. holds equity in Metabolon Inc., a metabolomics technologies company. This platform was used in the current analyses. R.K-D. formed Chymia LLC and PsyProtix, a Duke University biotechnology spinout aiming to transform the treatment of mental health disorders. JK, MArnold, and GK hold equity in Chymia LLC and IP in PsyProtix. 


\section{References}

1. Hampel, H. et al. Blood-based biomarkers for Alzheimer disease: mapping the road to the clinic. Nat Rev Neurol 14, 639-652 (2018).

2. Ross, C. A. \& Poirier, M. A. Protein aggregation and neurodegenerative disease. Nat. Med. 10, S10 (2004).

3. Arnold, M. et al. Sex and APOE epsilon4 genotype modify the Alzheimer's disease serum metabolome. Nat Commun 11, 1148 (2020).

4. Toledo, J. B. et al. Metabolic network failures in Alzheimer's disease: A biochemical road map. Alzheimer's Dement. 13, 965-984 (2017).

5. Mahajan, U. V. et al. Dysregulation of multiple metabolic networks related to brain transmethylation and polyamine pathways in Alzheimer disease: A targeted metabolomic and transcriptomic study. PLoS Med. 17, e1003012 (2020).

6. MahmoudianDehkordi, S. et al. Altered bile acid profile associates with cognitive impairment in Alzheimer's disease-An emerging role for gut microbiome. Alzheimers Dement 15, 76-92 (2019).

7. Nho, K. et al. Altered bile acid profile in mild cognitive impairment and Alzheimer's disease: Relationship to neuroimaging and CSF biomarkers. Alzheimers Dement 15, 232-244 (2019).

8. Kaddurah-Daouk, R. et al. Alterations in metabolic pathways and networks in Alzheimer's disease. Transl Psychiatry 3, e244 (2013).

9. Fuente, M. de la et al. Enzyme Therapy: Current Challenges and Future Perspectives. Int. J. Mol. Sci. 22, 9181 (2021).

10. Robertson, J. G. Enzymes as a special class of therapeutic target: clinical drugs and modes of action. Curr. Opin. Struct. Biol. 17, 674-679 (2007).

11. Bennett, D. A. et al. Overview and findings from the rush Memory and Aging Project. Curr Alzheimer Res 9, 646-663 (2012).

12. Bennett, D. A., Schneider, J. A., Arvanitakis, Z. \& Wilson, R. S. Overview and findings from the religious orders study. Curr Alzheimer Res 9, 628-645 (2012).

13. Krumsiek, J. et al. Gender-specific pathway differences in the human serum metabolome. Metabolomics 11, 1815-1833 (2015).

14. Stephens, C. R. et al. The Impact of Education and Age on Metabolic Disorders. Front. Public Heal. 8, 180 (2020).

15. Mittelstrass, K. et al. Discovery of Sexual Dimorphisms in Metabolic and Genetic Biomarkers. PLOS Genet. 7, e1002215 (2011).

16. Blusztajn, J. K., Lopez Gonzalez-Coviella, I., Logue, M., Growdon, J. H. \& Wurtman, R. J. Levels of phospholipid catabolic intermediates, glycerophosphocholine and glycerophosphoethanolamine, are elevated in brains of Alzheimer's disease but not of Down's syndrome patients. Brain Res 536, 240-244 (1990).

17. Krumsiek, J., Suhre, K., Illig, T., Adamski, J. \& Theis, F. J. Gaussian graphical modeling 
reconstructs pathway reactions from high-throughput metabolomics data. BMC Syst Biol 5, $21(2011)$.

18. Krumsiek, J. et al. Mining the Unknown: A Systems Approach to Metabolite Identification Combining Genetic and Metabolic Information. PLOS Genet. 8, e1003005 (2012).

19. Do, K. T. et al. Characterization of missing values in untargeted MS-based metabolomics data and evaluation of missing data handling strategies. Metabolomics 14, (2018).

20. Hardy, J. A. \& Higgins, G. A. Alzheimer's disease: The amyloid cascade hypothesis. Science (80-. ). 256, 184-185 (1992).

21. Hardy, J. \& Selkoe, D. J. The amyloid hypothesis of Alzheimer's disease: Progress and problems on the road to therapeutics. Science (80-. ). 297, 353-356 (2002).

22. Salloway, S. et al. Two Phase 3 Trials of Bapineuzumab in Mild-to-Moderate Alzheimer's Disease. N. Engl. J. Med. 370, 322-333 (2014).

23. Ostrowitzki, S. et al. A phase III randomized trial of gantenerumab in prodromal Alzheimer's disease. Alzheimer's Res. Ther. 9, (2017).

24. Honig, L. S. et al. Trial of Solanezumab for Mild Dementia Due to Alzheimer's Disease. N. Engl. J. Med. 378, 321-330 (2018).

25. Henley, D. et al. Preliminary Results of a Trial of Atabecestat in Preclinical Alzheimer's Disease. N. Engl. J. Med. 380, 1483-1485 (2019).

26. Egan, M. F. et al. Randomized Trial of Verubecestat for Prodromal Alzheimer's Disease. N. Engl. J. Med. 380, 1408-1420 (2019).

27. van der Kant, R., Goldstein, L. S. B. \& Ossenkoppele, R. Amyloid- $\beta$-independent regulators of tau pathology in Alzheimer disease. Nat. Rev. Neurosci. 21, 21-35 (2020).

28. Wang, W., Zhao, F., Ma, X., Perry, G. \& Zhu, X. Mitochondria dysfunction in the pathogenesis of Alzheimer's disease: recent advances. Mol. Neurodegener. 202015115 , 1-22 (2020).

29. Swerdlow, R. H. Mitochondria and Cell Bioenergetics: Increasingly Recognized Components and a Possible Etiologic Cause of Alzheimer's Disease. Antioxid. Redox Signal. 16, 1434 (2012).

30. Dematteis, G. et al. Proteomic analysis links alterations of bioenergetics, mitochondria-ER interactions and proteostasis in hippocampal astrocytes from 3xTg-AD mice. Cell Death Dis. $202011811,1-16$ (2020).

31. Sonntag, K.-C. et al. Late-onset Alzheimer's disease is associated with inherent changes in bioenergetics profiles. Sci. Reports 2017 71 7, 1-13 (2017).

32. Tynkkynen, J. et al. Association of branched-chain amino acids and other circulating metabolites with risk of incident dementia and Alzheimer's disease: A prospective study in eight cohorts. Alzheimer's Dement. 14, 723-733 (2018).

33. Monte, S. M. de la \& Wands, J. R. Alzheimer's Disease Is Type 3 Diabetes-Evidence Reviewed. J. diabetes Sci. Technol. 2, 1101 (2008).

34. Coon, K. D. et al. A High-Density Whole-Genome Association Study Reveals That APOE Is the Major Susceptibility Gene for Sporadic Late-Onset Alzheimer's Disease. J. Clin. 
Psychiatry 68, 0-0 (2007).

35. Corder, E. H. et al. Gene dose of apolipoprotein E type 4 allele and the risk of Alzheimer's disease in late onset families. Science (80-. ). 261, 921-923 (1993).

36. Husain, M. A., Laurent, B. \& Plourde, M. APOE and Alzheimer's Disease: From Lipid Transport to Physiopathology and Therapeutics. Front. Neurosci. 0, 85 (2021).

37. Saeed, A. et al. 7a-hydroxy-3-oxo-4-cholestenoic acid in cerebrospinal fluid reflects the integrity of the blood-brain barrier. J. Lipid Res. 55, 313 (2014).

38. Kerber, T. \& Vrielink, A. The role of hydrogen atoms in redox catalysis by the flavoenzyme cholesterol oxidase. Methods Enzymol. 634, 361-377 (2020).

39. MahmoudianDehkordi, S. et al. Altered bile acid profile associates with cognitive impairment in Alzheimer's disease-An emerging role for gut microbiome. Alzheimers Dement 15, 76-92 (2019).

40. Leng, F. \& Edison, P. Neuroinflammation and microglial activation in Alzheimer disease: where do we go from here? Nat. Rev. Neurol. 17, 157-172 (2021).

41. Borkowski, K. et al. Serum metabolomic biomarkers of perceptual speed in cognitively normal and mildly impaired subjects with fasting state stratification. Sci. Reports 2021111 11, 1-12 (2021).

42. Wu, G., Fang, Y. Z., Yang, S., Lupton, J. R. \& Turner, N. D. Glutathione Metabolism and Its Implications for Health. J. Nutr. 134, 489-492 (2004).

43. Allen, M. et al. Glutathione S-transferase omega genes in Alzheimer and Parkinson disease risk, age-at-diagnosis and brain gene expression: An association study with mechanistic implications. Mol. Neurodegener. 7, (2012).

44. Singhal, S. S. et al. Antioxidant role of glutathione S-transferases: 4-Hydroxynonenal, a key molecule in stress-mediated signaling. Toxicol. Appl. Pharmacol. 289, 361-370 (2015).

45. Dello, S. A. W. G. et al. Systematic review of ophthalmate as a novel biomarker of hepatic glutathione depletion. Clin. Nutr. 32, 325-330 (2013).

46. Snyder, N. W. et al. 15-oxoeicosatetraenoic acid is a 15-hydroxyprostaglandin dehydrogenase-derived electrophilic mediator of inflammatory signaling pathways. Chem. Biol. Interact. 234, 144 (2015).

47. Calder, P. C. Omega-3 Fatty Acids and Inflammatory Processes. Nutrients 2, 355 (2010).

48. Song, C., Li, X., Kang, Z. \& Kadotomi, Y. Omega-3 Fatty Acid Ethyl-Eicosapentaenoate Attenuates IL-1 $\beta$-Induced Changes in Dopamine and Metabolites in the Shell of the Nucleus Accumbens: Involved with PLA2 Activity and Corticosterone Secretion. Neuropsychopharmacol. 2007323 32, 736-744 (2006).

49. Wilson, C. S. \& Mongin, A. A. Cell Volume Control in Healthy Brain and Neuropathologies. Curr. Top. Membr. 81, 385-455 (2018).

50. Mueed, Z., Mehta, D., Rai, P. K., Kamal, M. A. \& Poddar, N. K. Cross-Interplay between Osmolytes and mTOR in Alzheimer's Disease Pathogenesis. Curr. Pharm. Des. 26, 4699_ 4711 (2020).

51. Street, T. O., Bolen, D. W. \& Rose, G. D. A molecular mechanism for osmolyte-induced 
protein stability. Proc. Natl. Acad. Sci. 103, 13997-14002 (2006).

52. Kushwah, N., Jain, V. \& Yadav, D. Osmolytes: A possible therapeutic molecule for ameliorating the neurodegeneration caused by protein misfolding and aggregation. Biomolecules 10, (2020).

53. Lauterborn, J. C. et al. Increased excitatory to inhibitory synaptic ratio in parietal cortex samples from individuals with Alzheimer's disease. Nat. Commun. 2021121 12, 1-15 (2021).

54. Bi, D., Wen, L., Wu, Z. \& Shen, Y. GABAergic dysfunction in excitatory and inhibitory (E/I) imbalance drives the pathogenesis of Alzheimer's disease. Alzheimer's Dement. 16, 13121329 (2020).

55. Yoon, J. H., Grandelis, A. \& Maddock, R. J. Dorsolateral Prefrontal Cortex GABA Concentration in Humans Predicts Working Memory Load Processing Capacity. J. Neurosci. 36, 11788-11794 (2016).

56. Wishart, D. S. et al. PathBank: A comprehensive pathway database for model organisms. Nucleic Acids Res. 48, D470-D478 (2020).

57. Wang, R. \& Reddy, P. H. Role of Glutamate and NMDA Receptors in Alzheimer's Disease. J. Alzheimer's Dis. 57, 1041-1048 (2017).

58. Handley, R. R. et al. Brain urea increase is an early Huntington's disease pathogenic event observed in a prodromal transgenic sheep model and HD cases. Proc. Natl. Acad. Sci. U. S. A. 114, E11293-E11302 (2017).

59. Ovsepian, S. V. \& O'Leary, V. B. Can Arginase Inhibitors Be the Answer to Therapeutic Challenges in Alzheimer's Disease? Neurotherapeutics 15, 1032-1035 (2018).

60. Yurdagul, A. et al. Macrophage Metabolism of Apoptotic Cell-Derived Arginine Promotes Continual Efferocytosis and Resolution of Injury. Cell Metab. 31, 518-533.e10 (2020).

61. Boada-Romero, E., Martinez, J., Heckmann, B. L. \& Green, D. R. The clearance of dead cells by efferocytosis. Nat. Rev. Mol. Cell Biol. 21, 398-414 (2020).

62. Hensley, K. \& Denton, T. T. Alternative functions of the brain transsulfuration pathway represent an underappreciated aspect of brain redox biochemistry with significant potential for therapeutic engagement. Free Radic. Biol. Med. 78, 123-134 (2015).

63. Kumar, R. Role of naturally occurring osmolytes in protein folding and stability. Arch Biochem Biophys 491, 1-6 (2009).

64. Mandal, P. K., Kansara, K. \& Dabas, A. The GABA-Working Memory Relationship in Alzheimer's Disease. J. Alzheimer's Dis. Reports 1, 43 (2017).

65. Jack, C. R. et al. Tracking pathophysiological processes in Alzheimer's disease: An updated hypothetical model of dynamic biomarkers. Lancet Neurol. 12, 207-216 (2013).

66. 2021 ALZHEIMER'S DISEASE FACTS AND FIGURES. Alzheimer's Association.

67. Holmes, M. V., Ala-Korpela, M. \& Smith, G. D. Mendelian randomization in cardiometabolic disease: Challenges in evaluating causality. Nat. Rev. Cardiol. 14, 577-599 (2017).

68. Hampel, H. et al. Precision pharmacology for Alzheimer's disease. Pharmacol. Res. 130, 331-365 (2018). 
69. Hampel, H. et al. Omics sciences for systems biology in Alzheimer's disease: State-of-theart of the evidence. Ageing Res. Rev. 69, (2021).

70. Olivier, M., Asmis, R., Hawkins, G. A., Howard, T. D. \& Cox, L. A. The need for multi-omics biomarker signatures in precision medicine. Int. J. Mol. Sci. 20, (2019).

71. Mostafavi, S. et al. A molecular network of the aging human brain provides insights into the pathology and cognitive decline of Alzheimer's disease. Nat. Neurosci. 21, 811-819 (2018).

72. Allen, M. et al. Human whole genome genotype and transcriptome data for Alzheimer's and other neurodegenerative diseases. Sci. Data 3, (2016).

73. Allen, M. et al. Conserved brain myelination networks are altered in Alzheimer's and other neurodegenerative diseases. Alzheimer's Dement. 14, 352-366 (2018).

74. Dieterle, F., Ross, A., Schlotterbeck, G. \& Senn, H. Probabilistic quotient normalization as robust method to account for dilution of complex biological mixtures. Application in1H NMR metabonomics. Anal. Chem. 78, 4281-4290 (2006).

75. Breunig, M. M., Kriegel, H.-P., Ng, R. T. \& Sander, J. LOF. Proc. 2000 ACM SIGMOD Int. Conf. Manag. data - SIGMOD '00 93-104 (2000) doi:10.1145/342009.335388.

76. Johnson, E. C. B. et al. Large-scale proteomic analysis of Alzheimer's disease brain and cerebrospinal fluid reveals early changes in energy metabolism associated with microglia and astrocyte activation. Nat. Med. 2020265 26, 769-780 (2020).

77. Chetnik, K. et al. maplet: An extensible R toolbox for modular and reproducible metabolomics pipelines. Bioinformatics

(2021) doi:10.1093/BIOINFORMATICS/BTAB741.

78. Benjamini, Y. \& Hochberg, Y. Controlling the False Discovery Rate: A Practical and Powerful Approach to Multiple Testing. J. R. Stat. Soc. Ser. B 57, 289-300 (1995).

79. Schäfer, J. \& Strimmer, K. A shrinkage approach to large-scale covariance matrix estimation and implications for functional genomics. Stat. Appl. Genet. Mol. Biol. 4, 1-30 (2005). 